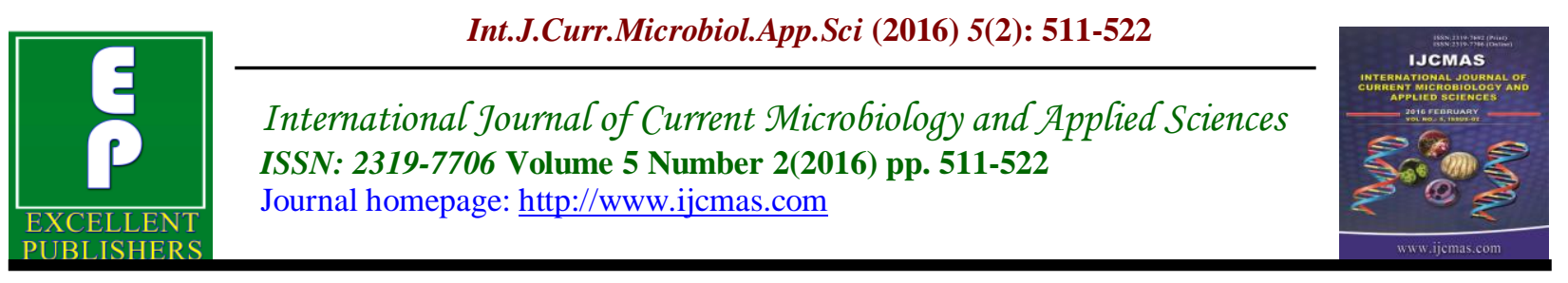

Original Research Article

doi: http://dx.doi.org/10.20546/ijcmas.2016.502.058

\title{
Synthesis, Characterization and Polymerization of 1,3,4- Oxadiazole Derivatives of Amoxicillin and Evaluation Antibacterial Activities
}

\author{
Entesar, Obeed. AL-Tamimi ${ }^{1}$ and Khalida, Ali.Thejeel ${ }^{2^{*}}$ \\ ${ }^{1}$ Department of Chemistry, College of Science, University of Baghdad, Iraq \\ ${ }^{2}$ Department of Pharmaceutical Chemistry, College of Pharmacy, \\ University of AL-Mustansriah, Iraq \\ *Corresponding author
}

Keywords

Amoxicillin, Acryl monomers, Antibacterial activity.

Article Info

Accepted:

26 January 2016

Available Online:

10, February 2016

\section{A B S T R A C T}

\section{Introduction}

Oxadiazole is five member heterocyclic compound containing an oxygen atom and two nitrogen atoms, oxadiazole have four isomers, 1,3,4-oxadiazole (1),1,2,4oxadiazole (2), 1,2,5-oxadiazole (3) and 1,2,3-oxadiazole (4), figure-1 (1).

Heterocyclic compounds containing 1,3,4oxadiazole are associated with diverse pharmacological activities such as antimicrobial (2), anti-intiflammatory (3), analgesic (4), anticancer (5), anticonvulsant (6). The rapid development of resistance to existing antimicrobial drugs generates a serious challenge to the scientific community.
The synthesis of new 1,3,4-oxadiazole derivatives and investigation of their biological behavior in last years has increase (7). In addition 1,3,4-oxadiazole have played a crucial part.in the development of theory in heterocyclic chemistry and also used extensively in organic chemistry (8-10).

Antimicrobial polymers class of insecticides are becoming increasingly important as alternative to the existing insecticides and in some cases even to the antibiotics. Mechanism action of large number of different polymers structurally often do not fully understand. However, it is known that some of them low potential of building 
microbial resistant strains (11).

Acrylate homo and copolymers are used in different applications such as films, coating, fibers, adhesives, filaments, printing inks, lacquers and binders (12-14). Homo and copolymers are a class of reactive polymers that used extensive fields (15).

\section{Experimental Section}

\section{Materials}

All chemicals used were of analytical reagent grade and they were available from Aldrich and Fluka Companies and amoxicillin trihydrate standard material was provided from state company for drug industries and medical appliance (SDI) Samaraa - Iraq.

\section{Instruments}

Melting points were determined in an open capillary tube and are uncorrected.

Infrared spectra were recorded in $\mathrm{KBr}$ on Shimadzu spectrophotometer.

The ${ }^{1}$ HNMR were measured in DMSO solutions on a Bruker-400 $\mathrm{MHz}$ spectrometer using TMS as internal reference (chemical shift in ppm).

All reactions was monitored by thin layer chromatography (TLC) and spots were visualized using iodine chamber.

The antibacterial activity was determined by Agar-well diffusion method.

\section{Synthesis Methods of Prepared Compounds}

Synthesis of methyl-6-(2-amino-2-(4hydroxyphenyl) acetamido)-2,2-dimethyl5-oxo-1-thia-4-azabicyclo(3.2.0) heptane3-carboxylate 2 (16).

In $250 \mathrm{~mL}$ round bottom flask was place a mixture of amoxicillin 1 (0.05mole) and an excess of absolute methanol $(150 \mathrm{~mL})$ with (5-7) drops of concentrate sulfuric acid. The mixture was refluxed for (3-4)hrs. The solution was cooled and poured into crushed ice. Precipitate obtained filtered, dried and recrystallized from ethanol.

Synthesis of 2-amino-N-((2acidhydrazide)-2,2-dimethyl-5-oxo-1-thia4-azabicyclo(3.2.0) heptan-6-yl)-2-(4hydroxy phenyl)acetamide 3 (17).

In $100 \mathrm{~mL}$ round bottom flask was place a mixture of (0.05mole) ester 2 , (0.05mole) hydrazine hydrate and $(50 \mathrm{~mL})$ ethanol refluxed for (5-8)hrs. The resultant mixture was concentrated, cooled and poured into crushed ice. The solid mass thus separated out was dried and recrystallized from ethanol.

Synthesis of 2-substituted-N-((2,2dimethyl-3-(benzyl ideneacidhydrazino)5-oxo-1-thia-4-azabicyclo(3.2.0) heptan-6yl)acet amide acid hydrazones (4-8) (18).

In $100 \mathrm{~mL}$ round bottom flask was place a solution of acid hydrazide 3 ( 0.01 mole) in methanol $(50 \mathrm{~mL})$ with different aliphatic and aromatic aldehyde (0.02mole) and (4-5) drops of glacial acetic acid as a catalyst. The mixture was refluxed for (3-6)hrs., the resultant allowed to cool and poured into cold water. Solid was collected after filtration and recrystallized from ethanol to give the pure product.

Synthesis of 2-substuted-N-2,2-dimethyl5-oxo-3-(5-phenyl)-1,3,4-oxadiazol-2 -yl)\}1-thia-4-azabicyclo (3.2.0)heptan-6yl)acetamides(9-13) (19).

To compounds (4-8) (0.01mole) were added glacial acetic acid $(40 \mathrm{~mL})$ and lead dioxide (0.01mole) with stirring to the homogenous 
solution. The mixture was stirred at $25^{\circ} \mathrm{C}$ for $1 \mathrm{hr}$. poured in to ice water $(100 \mathrm{ml})$ and left to stand for $24 \mathrm{hrs}$., filtered and recrystallized from ethanol.

Synthesis of acryl monomers of 2substuted-N-2,2-dimethyl-5-oxo-3-(5phenyl)-1,3,4-oxadiazol-2 -yl)\}-1-thia-4azabicyclo (3.2.0)heptan-6-yl)acetamides (14-18) (20).

In $50 \mathrm{~mL}$ round bottom flask dissolve (0.002mole) of hydrazones(9-13) in THF $(5 \mathrm{~mL})$ with $(0.002$ mole $)$ of $\mathrm{Et}_{3} \mathrm{~N}$ and (0.002mole) and acryloyl chloride in THF $(5 \mathrm{~mL})$ was added drop wise with stirring at $0^{\circ} \mathrm{C}$ for (4-6)hrs. The $\mathrm{Et}_{3} \mathrm{~N}-\mathrm{HCl}$ salt was precipitated and filtered. The filterate was poured with stirring into $(100 \mathrm{~mL})$ water to precipitate the product, filtered and recrystallization from ethanol.

Synthesis of acryl polymers of 2substuted-N-2,2-dimethyl-5-oxo-3-(5phenyl)- $\quad$ 1,3,4-oxadiazol-2-yl)-1-thia-4azabicyclo (3.2.0) heptan-6-yl) acetamides (19-23) (21).

In a screw-capped polymerization bottle dissolve (0.001mole) of monomers (14-18) in $(5 \mathrm{~mL}) \mathrm{THF}, \mathrm{DMF}$ or DMSO. An amount equal to $0.02 \%$ of the monomers wt. of AIBN added. bottle was flushed with nitrogen gas about few min. and firmly stoppered. The maintained at $(70-80)^{\circ} \mathrm{C}$ in constant temperature water bath for (1-2)hrs. Then the solution was poured into about $50 \mathrm{~mL}$ of water or methanol. The precipitate was collected by filterate, washed with methanol several time and dried

\section{Results and Discussion}

In the current study a novel series of amoxicillin based on 1,3,4- oxadiazoles were synthesized using compound 1 (amoxicillin) as starting material for synthesis. the synthesis of compounds (2-23) were performed according to the outline given in scheme-1.

Compounds (9-13) were prepared by treatment compounds(4-8) with lead dioxide in glacial acetic acid affords intramolecular cyclization to give 1,3,4-oxadiazoled,then reacted with acryloly chloride to give drug containing acryl monomers (14-18). The polymerization of monomers were carried out in DMF, using AIBN as initiator. physical properties for compounds(2-23) are listed in table -1

The structures of all compounds were confirmed by FT-IR and ${ }^{1}$ HNMR spectroscopy that showed absorption bands at (3221-3283), (1609-1626), (1742-1758) and (1659-1686) $\mathrm{cm}^{-1}$ regions, confirming the presence of $v(\mathrm{~N}-\mathrm{H}), v(\mathrm{CH}=\mathrm{N}), v(\mathrm{C}=\mathrm{O})$ azetidinone and $v(\mathrm{C}=\mathrm{O})$ amide respectively The FT-IR spectrum of compound 2 characteristic absorption bands at $(3526,3462)$ and $1740 \mathrm{~cm}^{-1}$ for $v\left(\mathrm{NH}_{2}\right)$ and $v(\mathrm{C}=\mathrm{O})$ ester respectively, the absorption bands of $v(\mathrm{C}=\mathrm{O})$ and $v\left(\mathrm{NH}_{2}\right)$ disappearance in compound 3 and appearance absorption bands at $(1607-1622) \mathrm{cm}^{-1}$ for $v(\mathrm{CH}=\mathrm{N})$ of compounds (4-8). FT-IR spectra of the compounds (9-13) showed absorption bands at (1224-1242) $\mathrm{cm}^{-1}$ confirming the presence $v$ (C-O-C), while the compounds (14-18) appearance absorption bands at (1695-1702) $\mathrm{cm}^{-1}$ for $v(\mathrm{C}=\mathrm{O})$ imide and at (1604-1614) $\mathrm{cm}^{-1}$ for $v(C=C)$ olefinic that disappearance in compounds (19-23) due to polymerized, all details FT-IR spectral data for compounds are listed in table -2 .

The chemical shifts in the ${ }^{1}$ HNMR spectra of the respective derivatives verified their structures. The spectra of compounds (2-23) showed the characteristic protons of benzene, 2- $\mathrm{CH}_{3}, \mathrm{CH}-\underline{\mathrm{CH}}-\mathrm{S}, \mathrm{CH}-\mathrm{CH}-\mathrm{S}$ and $\mathrm{OH}$ phenolic at around $\boldsymbol{\delta}(6.51-7.95),(1.35-$ 1.55), (4.83-4.89)., (5.16-5.34) and (5.32- 
5.39) ppm respectively. The ${ }^{1} \mathrm{HNMR}$ spectra of compound 2 exhibited characteristic $3 \mathrm{H}$ protons of $\mathrm{COOCH}_{3}$ at $\delta 3.68 \mathrm{ppm}$ and the characteristic $1 \mathrm{H}$ proton of $\delta-\mathrm{CHCOOCH}_{3}$ at $\delta 4.68 \mathrm{ppm}$. ${ }^{1} \mathrm{HNMR}$ spectra of compound 3 exhibited characteristic $3 \mathrm{H}$ protons of $\mathrm{NH}-$ $\mathrm{NH}_{2}, 1 \mathrm{H}$ of $\mathrm{NH}-\mathrm{NH}_{2}$, and $\mathrm{CH}-\mathrm{NH}_{2}$ at $\delta 2.01$, at $\delta 8.02 \mathrm{ppm}$ and $\delta 4.81 \mathrm{ppm}$ respectively. 1HNMR spectra of compounds $(4,5)$ exhibited characteristic $1 \mathrm{H}$ proton of $\mathrm{CH}-$ $\mathrm{N}=\mathrm{CH}$ and $1 \mathrm{H}$ proton of $\mathrm{NH}-\mathrm{N}=\underline{\mathrm{CH}}$ at around $\delta(8.13-8.26)$ and at around $\delta(8.48$ 8.73) $\mathrm{ppm}$ respectively. 1HNMR spectra of compounds $(9,10)$ exhibited characteristic
$1 \mathrm{H}$ proton of $\mathrm{CH}-\mathrm{N}=\mathrm{CH}$ and $1 \mathrm{H}$ proton of $\mathrm{NH}$-amide around $\delta 5.34$ and at $\delta(8.03$ 8.09) pp respectively. 1HNMR spectrum of compound 14 exhibited characteristic $1 \mathrm{H}$ proton of $\mathrm{CH}-\mathrm{Oxa}, 2 \mathrm{H}$ protons of $\mathrm{CH}_{2}=\mathrm{CH}$ and $1 \mathrm{H}$ proton $\mathrm{CH}_{2}=\underline{\mathrm{CH}}$ at $\delta 5.72 \mathrm{ppm}$, at $\delta$ $4.68 \mathrm{ppm}$ and at $\delta 6.268 \mathrm{ppm}$, while 1HNMR spectrum of compound 19 exhibited characteristic $2 \mathrm{H}$ protons of $\left(\mathrm{CH}_{2} \mathrm{CH}-\right)_{\mathrm{n}^{-}}$and $1 \mathrm{H}$ proton of $-\left(\mathrm{CH}_{2}-\mathrm{CH}-\right)_{\mathrm{n}^{-}}$ at $\delta 1.657 \mathrm{ppm}$ and at $\delta 2.615 \mathrm{ppm}$ respectively. All details ${ }^{1} \mathrm{HNMR}$ spectral data are listed in table-3, figures (2-9).

Table.1 Physical Properties of Synthesized Compounds(2-23)

\begin{tabular}{|c|c|c|c|c|}
\hline \multirow[b]{2}{*}{$\begin{array}{l}\text { Comp. } \\
\text { Code }\end{array}$} & \multicolumn{4}{|c|}{ Physical properties } \\
\hline & Compound structure & Compound name & $\begin{array}{l}\text { Dec.p } \\
{ }^{\mathrm{O}} \mathrm{C}\end{array}$ & $\begin{array}{l}\text { Yield } \\
\%\end{array}$ \\
\hline 2 & & $\begin{array}{l}\text { Methyl-6-(2-amino-2-(4-hydroxyphenyl)acetamido)-2,2- } \\
\text { dimethyl-5-oxo-1-thia-4-azabicyclo(3.2.0)heptane-3- } \\
\text { carboxylate }\end{array}$ & 196 & 87 \\
\hline 3 & & $\begin{array}{l}\text { 2-amino-N-((2-acidhydrazide)-2,2-dimethyl-5-oxo-1-thia-4- } \\
\text { azabicyclo(3.2.0)heptan-6-yl)-2-(4-hydroxy phenyl)acetamide }\end{array}$ & 184 & 89 \\
\hline 4 & & $\begin{array}{l}\text { 2-((benzylidene amino)-(4-hydroxy phenyl))-N-((2,2-dimethyl- } \\
\text { 3-(benzyl ideneacidhydrazino)-5-oxo-1-thia-4-azabicyclo(3.2.0) } \\
\text { heptan-6-yl)acet amide }\end{array}$ & 170 & 75 \\
\hline 5 & & $\begin{array}{l}\text { 2-((2-hydroxy benzylidene amino) (4-hydroxyphenyl)) -N-(2,2- } \\
\text { dimethyl-2 -hydroxybenzyl ideneacid)hydrazine-5-oxo-1-thia- } \\
\text { 4-azabicyclo(3.2.0) heptan-6-yl) acetamide }\end{array}$ & 172 & 85 \\
\hline 6 & & $\begin{array}{l}\text { N-((2,2-dimethyl-3-(nitrobenzyl ideneacidhydrazino)-5-oxo-1- } \\
\text { thia-4-azabicyclo(3.2.0)heptan-6-yl))-2-(4-hydroxyphenyl)-2- } \\
\text { ((4-nitrobenzyl idene)amino) acetamide }\end{array}$ & 166 & 71 \\
\hline 7 & & $\begin{array}{l}\text { N-((2,2-dimethyl-3-(2-naphthalen-2-ylmethyleneacid } \\
\text { hydrazineco)-5-oxo-1-thia-4-aza bicyclo(3.2.0) heptan-6-yl))-2- } \\
\text { ((4-hydroxyphenyl) } \\
\text { ylmethylene)amino)acetamide }\end{array}$ & 176 & 89 \\
\hline 8 & & $\begin{array}{l}\text { N-((2,2-dimethyl-5-oxo-2-(2-propyl ideneacidhydrazino)-1-thia- } \\
\text { 4-aza bicyclo(3.2.0)heptan-6-yl))-2-((4-hydroxyphenyl)-3- } \\
\text { (propylideneamino))acetamide }\end{array}$ & 160 & 68 \\
\hline
\end{tabular}




\begin{tabular}{|c|c|c|c|c|}
\hline 9 & 5 & $\begin{array}{l}\text { 2-(\{benzylideneamino)(4-hydroxyphenyl)\}-N-2,2-dimethyl-5- } \\
\text { oxo-3-(5-phenyl)-1,3,4-oxadiazol-2 } \\
\begin{array}{ll}\text { (3.2.0)heptan-6-yl)acetamide } & \end{array}-1 \text {-thia-4-azabicyclo } \\
\end{array}$ & 154 & 86 \\
\hline 10 & & $\begin{array}{l}\text { 2-((2-hydroxybenzylidene )amino)(4-hydroxyphenyl ))-N-(3-(5- } \\
\text { (2-hydroxy phenyl)-1,3,4-oxadiazol-2-yl)-2,2-dimethyl-5-oxo- } \\
\text { 1-thia-4-azabicyclo(3.2.0) heptan-6-yl)acetamide }\end{array}$ & 160 & 75 \\
\hline 11 & & $\begin{array}{l}\mathrm{N} \text {-(2,2-dimethyl-3-(5-(4-nitrophenyl)-1,3,4-oxadiazol-2-yl)-5- } \\
\text { oxo-1-thia-4-azabicyclo(3.2.0) heptan-6-yl)-2-((4- } \\
\text { hydroxyphenyl)(4-nitrobenzylidene)amino)acetamide }\end{array}$ & 158 & 85 \\
\hline 12 & & $\begin{array}{l}\mathrm{N}-(2,2-\text { dimethyl-3-(5-(naphthalen-2-yl)-1,3,4-oxadiazol-2-yl)-5- } \\
\text { oxo-1-thia-4-azabicyclo(3.2.0) heptan-6-yl)-2-((4- } \\
\text { hydroxyphenyl)(2-naphthalen-2-ylmethylene )amino) acetamide }\end{array}$ & 166 & 76 \\
\hline 13 & & $\begin{array}{l}\text { N-3-(5-ethyl-1,3,4-oxadiazol-2-yl)-2,2-dimethyl-5-oxo-1-thia-4- } \\
\text { azabicyclo(3.2.0)heptan-6-yl)-2-((4- } \\
\text { hydroxyphenyl)(propylideneamino))acetamide }\end{array}$ & 140 & 73 \\
\hline 14 & & $\begin{array}{l}\mathrm{N}-((2-\{\text { (benzylidene amino)(4-hydroxy phenyl) }\} \text { acetyl)-N-\{2,2- } \\
\text { dimethyl-3-(5-phenyl-1,3,4-oxadiazol-2-yl)-5-oxo } \\
\text { azabicyclo (3.2.0)heptan-6-yl }\} \text { acrylamide }\end{array}$ & 162 & 53 \\
\hline 15 & & $\begin{array}{l}\mathrm{N}-((2-\{(2-h y d r o x y b e n z y l i d e n e) \text { amino }) \text { (4-hydroxyphenyl })\} \\
\text { acetyl)-N-\{3-(5-(2-hydroxyphenyl-1,3,4-oxadiazol-2-yl) }\}-\{2,2- \\
\text { dimethyl-5-oxo-1-thia-4-azabicyclo(3.2.0)heptan-6-yl }\} \\
\text { acrylamide }\end{array}$ & 158 & 61 \\
\hline 16 & & $\begin{array}{l}\mathrm{N} \text {-(2,2-dimethyl-3-(5-(4-nitrophenyl)-1,3,4-oxadiazol-2-yl)-5- } \\
\text { oxo -1-thia-4-azabicyclo } \quad(3.2 .0) \text { heptan-6-yl)-N-(2-\{(4- } \\
\text { hydroxyphenyl ) ((4-nitrobenzylidene) amino }\} \text { acetyl }) \\
\text { acrylamide }\end{array}$ & 160 & 58 \\
\hline 17 & & $\begin{array}{l}\mathrm{N} \text {-\{2,2-dimethyl-3-(5-(naphthalen-2-yl)-1,3,4-oxadiazol-2-yl }\}- \\
5 \text {-oxo-1-thia-4-azabicyclo(3.2.0)heptan-6-yl)-N-(2-\{(4- } \\
\text { hydroxyphenyl) } \\
\text { ylmethylene)amino }\} \text { acetyl)acrylamide }\end{array}$ & 166 & 66 \\
\hline 18 & & $\begin{array}{l}\mathrm{N}-(\{3-(5-\text {-ethyl-1,3,4-oxadiazol-2-yl }\}-2,2-\text { dimethyl-5-oxo-1- } \\
\text { thia-4-azabicyclo (3.2.0)heptan-6-yl)-N-(2-\{(4-hydroxyphenyl ) } \\
\text { (propylideneamino\} acetyl)acrylamide }\end{array}$ & 156 & 59 \\
\hline 19 & & $\begin{array}{l}\text { Poly(N-((2-\{( benzylideneamino)(4-hydroxyphenyl) }\} \text { acetyl)-N- } \\
\{2,2 \text {-dimethyl-3-(5-phenyl-1,3,4-oxa diazol-2-yl)-5-oxo-1-thia- } \\
\text { 4-azabicyclo (3.2.0)heptan-6-yl }\} \text { acrylamide })\end{array}$ & Oily & 70 \\
\hline
\end{tabular}




\begin{tabular}{|c|c|c|c|c|}
\hline 20 & & $\begin{array}{l}\text { Poly(N-((2-\{(2-hydroxy benzylidene)amino)(4-hydroxyphenyl) } \\
\text { \}acetyl)-N-\{3-(5-(2-hydroxyphenyl)-1,3,4-oxadiazol-2-yl })\}- \\
\{2,2 \text {-dimethyl-5-oxo-1-thia-4-azabicyclo(3.2.0) heptan-6-yl }\} \\
\text { acrylamide) }\end{array}$ & Oily & 65 \\
\hline 21 & & 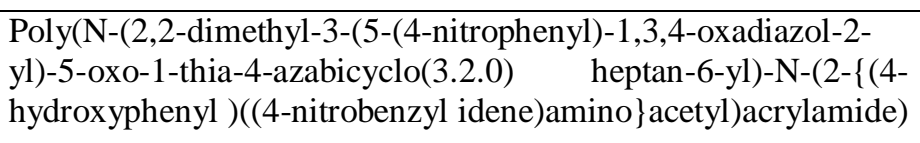 & 132 & 71 \\
\hline 22 & & 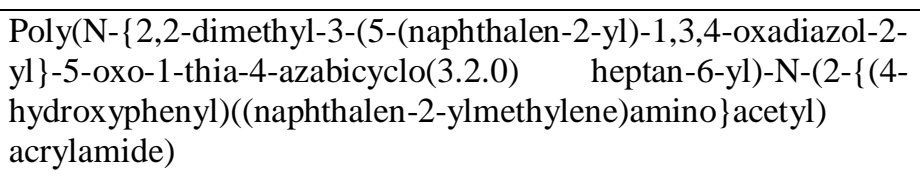 & Oily & 69 \\
\hline 23 & & $\begin{array}{l}\text { Poly(N-(\{3-(5-ethyl-1,3,4-oxadiazol-2-yl }\}-2,2 \text {-dimethyl-5-oxo- } \\
\text { 1-thia-4-azabicyclo(3.2.0)heptan-6-yl)-N-(2-\{(4-hydroxyhenyl) } \\
\text { (propylideneamino\}acetyl) acrylamide) }\end{array}$ & Oily & 62 \\
\hline
\end{tabular}

Table.2 Ft-Ir Spectral Data of Synthesized Compounds(2-23)

\begin{tabular}{|c|c|c|c|c|c|c|c|c|c|}
\hline \multirow[b]{2}{*}{$\begin{array}{l}\text { Com } \\
\text { p. } \\
\text { Code }\end{array}$} & \multicolumn{6}{|c|}{ Major FT-IR absorption $\mathrm{cm}^{-1}$} & \multirow[b]{2}{*}{ 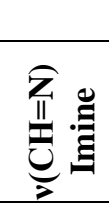 } & \multirow[b]{2}{*}{ 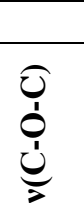 } & \multirow[b]{2}{*}{ 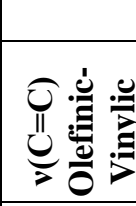 } \\
\hline & 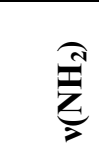 & 究递 & 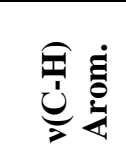 & 承 & 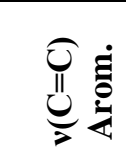 & $\begin{array}{l}\quad \quad v(C=O) \\
\text { 1.azetidinone } \\
\text { 2.ester(imide) } \\
\text { 3.amide }\end{array}$ & & & \\
\hline 2 & $\begin{array}{l}3526 \\
3462\end{array}$ & 3169 & 3042 & $\begin{array}{l}2970 \\
2936\end{array}$ & $\begin{array}{l}1584 \\
1518\end{array}$ & $\begin{array}{l}1.1776 \\
2.1740 \\
3.1688\end{array}$ & - & - & - \\
\hline 3 & $\begin{array}{l}3527 \\
3443\end{array}$ & 3219 & 3053 & $\begin{array}{l}2969 \\
2930\end{array}$ & $\begin{array}{l}1599 \\
1514\end{array}$ & $\begin{array}{l}\text { 1. } 1770 \\
2 .- \\
3.1668\end{array}$ & - & - & - \\
\hline 4 & - & 3233 & 3032 & $\begin{array}{l}2967 \\
2930\end{array}$ & $\begin{array}{l}1585 \\
1514\end{array}$ & $\begin{array}{l}1.1742 \\
2 .- \\
3.1667\end{array}$ & 1614 & - & - \\
\hline 5 & - & 3215 & 3048 & $\begin{array}{l}2970 \\
2930\end{array}$ & $\begin{array}{l}1578 \\
1514\end{array}$ & $\begin{array}{l}1.1744 \\
2 .- \\
3.1665\end{array}$ & 1622 & - & - \\
\hline 6 & - & 3241 & 3080 & $\begin{array}{l}2970 \\
2932\end{array}$ & $\begin{array}{l}1587 \\
1520\end{array}$ & $\begin{array}{l}1.1752 \\
2 .- \\
3.1688\end{array}$ & 1607 & - & - \\
\hline 7 & - & 3221 & 3055 & $\begin{array}{l}2969 \\
2928\end{array}$ & $\begin{array}{l}1586 \\
1514\end{array}$ & $\begin{array}{l}1.1748 \\
2 .- \\
3.1680\end{array}$ & 1613 & - & - \\
\hline 8 & - & 3229 & 3044 & $\begin{array}{l}2970 \\
2932\end{array}$ & $\begin{array}{l}1590 \\
1516\end{array}$ & $\begin{array}{l}1.1758 \\
2 .- \\
3.1659\end{array}$ & 1613 & - & - \\
\hline 9 & - & 3221 & 3030 & $\begin{array}{l}2951 \\
2931\end{array}$ & $\begin{array}{l}1585 \\
1518\end{array}$ & $\begin{array}{l}1.1742 \\
2 .- \\
3.1670\end{array}$ & 1626 & 1236 & - \\
\hline 10 & - & 3262 & 3033 & $\begin{array}{l}2972 \\
2932\end{array}$ & $\begin{array}{l}1576 \\
1514\end{array}$ & $\begin{array}{l}1.1744 \\
2 .- \\
3.1659\end{array}$ & 1616 & 1242 & - \\
\hline
\end{tabular}


Int.J.Curr.Microbiol.App.Sci (2016) 5(2): 511-522

\begin{tabular}{|c|c|c|c|c|c|c|c|c|c|}
\hline 11 & - & 3283 & 3077 & $\begin{array}{l}2967 \\
2930\end{array}$ & $\begin{array}{l}1588 \\
1520\end{array}$ & $\begin{array}{l}1.1752 \\
2 .- \\
3.1686\end{array}$ & 1609 & 1234 & - \\
\hline 12 & - & 3268 & 3055 & $\begin{array}{l}2967 \\
2929\end{array}$ & $\begin{array}{l}1596 \\
1512\end{array}$ & $\begin{array}{l}1.1748 \\
2 .- \\
3.1684\end{array}$ & 1613 & 1229 & - \\
\hline 13 & - & 3269 & 3063 & $\begin{array}{l}2969 \\
2932\end{array}$ & $\begin{array}{l}1590 \\
1524\end{array}$ & $\begin{array}{l}1.1758 \\
2 .- \\
3.1686 \\
\end{array}$ & 1613 & 1224 & - \\
\hline 14 & - & - & 3063 & $\begin{array}{l}2970 \\
2928\end{array}$ & $\begin{array}{l}1543 \\
1514\end{array}$ & $\begin{array}{l}1.1756 \\
2 .(1695) \\
3.1655\end{array}$ & 1620 & 1229 & 1614 \\
\hline 15 & - & - & 3078 & $\begin{array}{l}2959 \\
2928\end{array}$ & $\begin{array}{l}1578 \\
1514\end{array}$ & $\begin{array}{l}1.1765 \\
2 .(1702) \\
3.1653\end{array}$ & 1617 & 1231 & 1604 \\
\hline 16 & - & - & 3073 & $\begin{array}{l}2963 \\
2930\end{array}$ & $\begin{array}{l}1598 \\
1520\end{array}$ & $\begin{array}{l}1.1759 \\
2 .(1698) \\
3.1659 \\
\end{array}$ & 1611 & 1225 & 1606 \\
\hline 17 & - & $=$ & 3071 & $\begin{array}{l}2957 \\
2928\end{array}$ & $\begin{array}{l}1596 \\
1510\end{array}$ & $\begin{array}{l}1.1766 \\
2 .(1701) \\
3.1657 \\
\end{array}$ & 1611 & 1226 & 1607 \\
\hline 18 & - & - & 3063 & $\begin{array}{l}2969 \\
2932\end{array}$ & $\begin{array}{l}1590 \\
1524\end{array}$ & $\begin{array}{l}1.1748 \\
2 .(1697) \\
3.1659\end{array}$ & 1612 & 1227 & 1605 \\
\hline 19 & - & - & 3063 & $\begin{array}{l}2965 \\
2922\end{array}$ & $\begin{array}{l}1541 \\
1514\end{array}$ & $\begin{array}{l}1.1744 \\
2 .(1672)\end{array}$ & 1605 & 1231 & \\
\hline 20 & - & - & 3069 & $\begin{array}{l}2967 \\
2924\end{array}$ & $\begin{array}{l}1573 \\
1516\end{array}$ & $\begin{array}{l}1.1745 \\
2 .(1655)\end{array}$ & 1613 & 1236 & \\
\hline 21 & - & - & 3073 & $\begin{array}{l}2967 \\
2926 \\
\end{array}$ & $\begin{array}{l}1599 \\
1520 \\
\end{array}$ & $\begin{array}{l}1.1765 \\
2 .(1676)\end{array}$ & 1619 & 1236 & \\
\hline 22 & - & $=$ & 3057 & $\begin{array}{l}2965 \\
2924\end{array}$ & $\begin{array}{l}1596 \\
1512\end{array}$ & $\begin{array}{l}1.1759 \\
2 .(1682)\end{array}$ & 1609 & 1226 & \\
\hline 23 & - & - & 3064 & $\begin{array}{l}2960 \\
2924 \\
\end{array}$ & $\begin{array}{l}1590 \\
1513 \\
\end{array}$ & $\begin{array}{l}1.1764 \\
2 .(1643) \\
\end{array}$ & 1611 & 1224 & \\
\hline
\end{tabular}

Figure.1
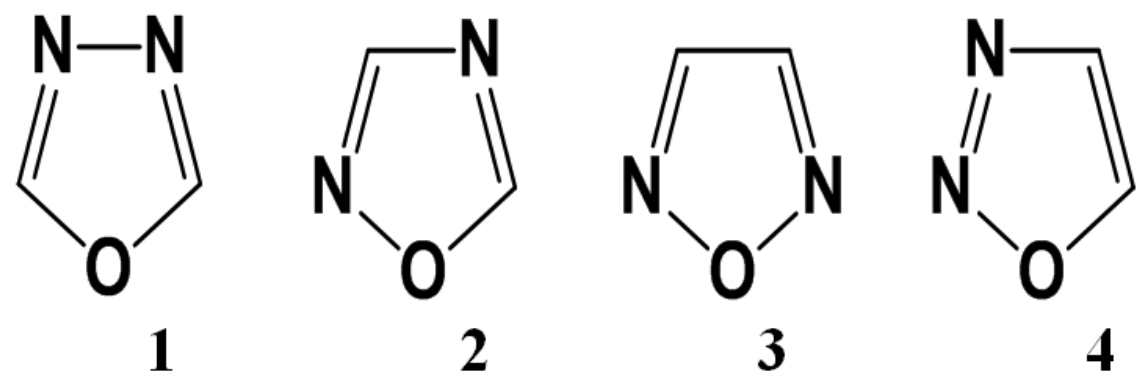
Table.3 1HNMRspectral Data of Selected Synthesized Compounds

\begin{tabular}{|c|c|}
\hline $\begin{array}{c}\text { Comp. } \\
\text { No. }\end{array}$ & ${ }^{1}$ HNMR Spectral data $(\delta$ ppm $)$ \\
\hline 2 & $\begin{array}{l}\text { 6.63-7.37(m,4H,Ar-H }) ; 1.55\left(\mathrm{~s}, 6 \mathrm{H}, 2 \mathrm{CH}_{3}\right) ; 3.68\left(\mathrm{~s}, 3 \mathrm{H},-\mathrm{COOCH}{ }_{3}\right) ; 4.68\left(\mathrm{~s}, 1 \mathrm{H},-\mathrm{N}-\underline{\mathrm{CH}}-\mathrm{COOCH}_{3}\right) ; \\
\left.\text { 4.75(s,1H,- } \mathrm{CH}-\mathrm{NH}_{2}\right) ; 4.82(\mathrm{~d}, 1 \mathrm{H}, \mathrm{CH}-\mathrm{CH}-\mathrm{S}) \text { Azet; } 5.30\left(\mathrm{~s}, 2 \mathrm{H},-\underline{\mathrm{NH}}_{2}\right) ; 5.34(\mathrm{~d}, 1 \mathrm{H}, \underline{\mathrm{CH}}-\mathrm{CH}-\mathrm{S}) \text { Azet.; } \\
\text { 5.35(s,1H,-OH}) ; 8.06(\mathrm{~s}, 1 \mathrm{H},-\mathrm{NH} \text { amide). }\end{array}$ \\
\hline 3 & 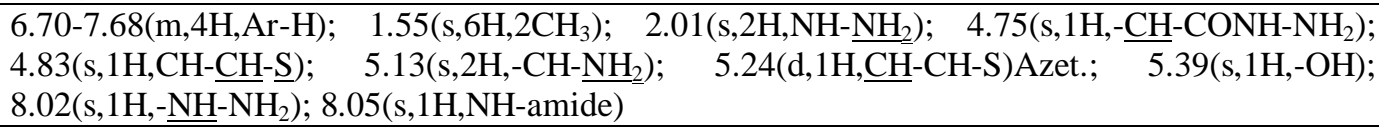 \\
\hline 4 & 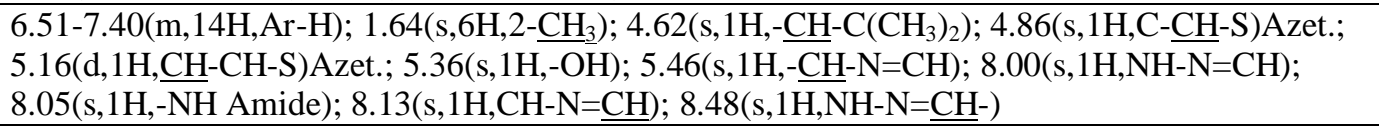 \\
\hline 5 & 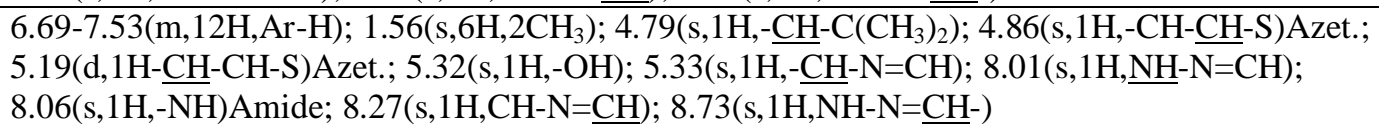 \\
\hline 9 & 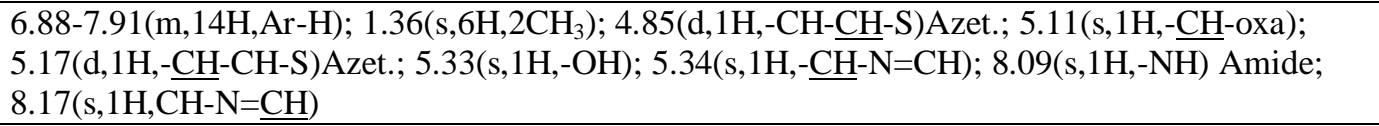 \\
\hline 10 & 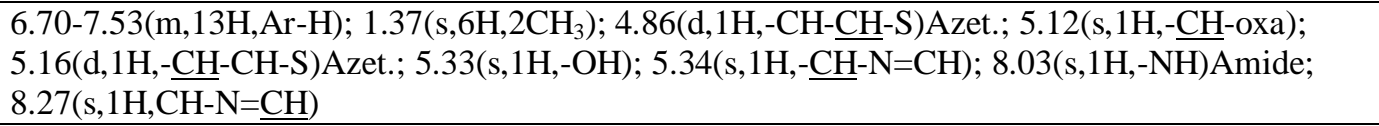 \\
\hline 14 & $\begin{array}{l}\text { 7.175-7.778(m,14H,Ar-H }) ; \quad 1.354\left(\mathrm{~s}, 6 \mathrm{H}, 2 \mathrm{CH}_{3}\right) ; \quad 4.897(\mathrm{~d}, 1 \mathrm{H},-\mathrm{CH}-\mathrm{CH}-\mathrm{S}) \mathrm{Azet} ; \quad 5.146(\mathrm{~s}, 1 \mathrm{H},-\mathrm{CH}- \\
\text { Oxa); 5.196(d,1H,-CH-CH-S)Azet; 5.386(s,1H,OH phenolic); } 5.455(\mathrm{~s}, 1 \mathrm{H}, \mathrm{CH}-\mathrm{N}=\mathrm{CH}) ; \\
5.720\left(\mathrm{~s}, 2 \mathrm{H},-\mathrm{CH}_{2}=\mathrm{CH}-\right) ; 6.268\left(\mathrm{~s}, 1 \mathrm{H}, \mathrm{CH}_{2}=\mathrm{CH}-\right) ; 8.014(\mathrm{~s}, 1 \mathrm{HCH},-\mathrm{N}=\mathrm{CH})\end{array}$ \\
\hline 19 & $\begin{array}{l}6.925-7.957(\mathrm{~m}, 14 \mathrm{H}, \mathrm{Ar}-\mathrm{H}) ; 1.392\left(\mathrm{~s}, 6 \mathrm{H}, 2 \mathrm{CH}_{3}\right) ; 1.657\left(\mathrm{~d}, 2 \mathrm{H},-\left(-\mathrm{CH}_{2}-\mathrm{CH}-\right) \mathrm{n}-\right) ; 2.615\left(\mathrm{t}, 1 \mathrm{H},-\left(-\mathrm{CH}_{2}-\right.\right. \\
\mathrm{CH}-) \mathrm{n}-) ; \quad 4.847(\mathrm{~d}, 1 \mathrm{H},-\mathrm{CH}-\mathrm{CH}-\mathrm{S}) \mathrm{Azet} ; \quad 5.145(\mathrm{~s}, 1 \mathrm{H},-\mathrm{CH}-\mathrm{Oxa}) ; \quad 5.202(\mathrm{~d}, 1 \mathrm{H},-\mathrm{CH}-\mathrm{CH}-\mathrm{S}) \text { Azet; } \\
5.352(\mathrm{~s}, 1 \mathrm{H}, \mathrm{OH} \text { phenolic); } 5.385(\mathrm{~s}, 1 \mathrm{H}, \mathrm{CH}-\mathrm{N}=\mathrm{CH}) ; 8.226(\mathrm{~s}, 1 \mathrm{HC}-\mathrm{H},-\mathrm{N}=\mathrm{CH})\end{array}$ \\
\hline
\end{tabular}

Table.4 Antibacterial Activities of Compounds (9-13) and (19-23)

\begin{tabular}{|l|l|l|l|l|}
\hline \multirow{2}{*}{\begin{tabular}{l} 
Comp. No. \\
\cline { 2 - 5 }
\end{tabular}} & $\begin{array}{c}\text { Staphylococcus } \\
\text { aurous }\end{array}$ & Bacillus subtitles' & Escherichia coli & $\begin{array}{c}\text { Pseudomonas } \\
\text { Aeruginosa }\end{array}$ \\
\hline $\mathbf{9}$ & 15 & 11 & 9 & 5 \\
\hline $\mathbf{1 0}$ & 14 & 12 & 8 & - \\
\hline $\mathbf{1 1}$ & 15 & 11 & 9 & 4 \\
\hline $\mathbf{1 2}$ & 14 & 12 & 8 & - \\
\hline $\mathbf{1 3}$ & 16 & 13 & 5 & 6 \\
\hline $\mathbf{1 9}$ & 16 & 13 & 7 & 5 \\
\hline $\mathbf{2 0}$ & 15 & 12 & 6 & - \\
\hline $\mathbf{2 1}$ & 16 & 13 & 8 & 5 \\
\hline $\mathbf{2 2}$ & 16 & 14 & 7 & - \\
\hline $\mathbf{2 3}$ & 14 & 12 & 6 & - \\
\hline Amoxicillin(A) & 14 & 11 & 5 & - \\
\hline DMSO & - & - & - & - \\
\hline
\end{tabular}


Figure.2 1HNMR Spectrum for Compound(2)

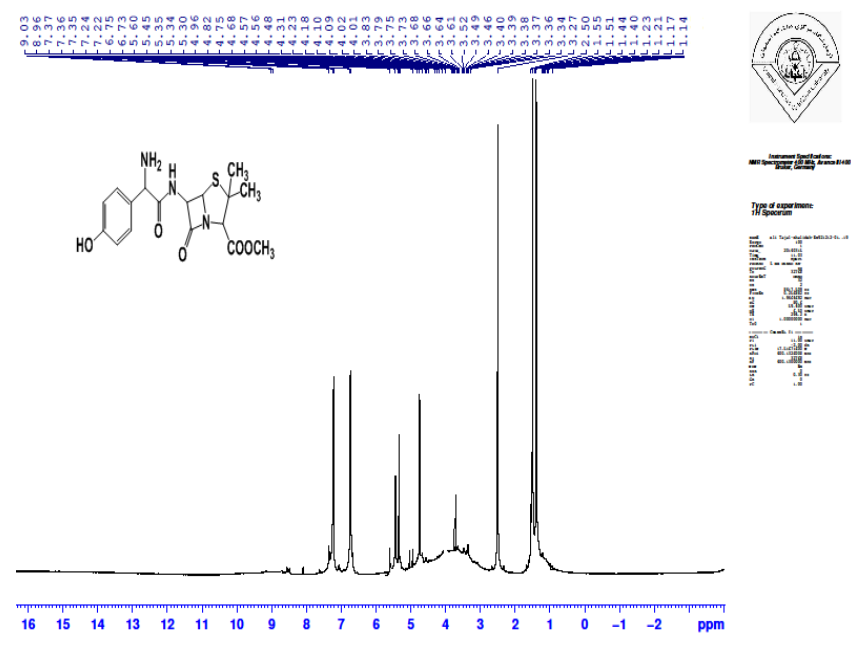

Figure.3 1HNMR Spectrum for Compound(3)

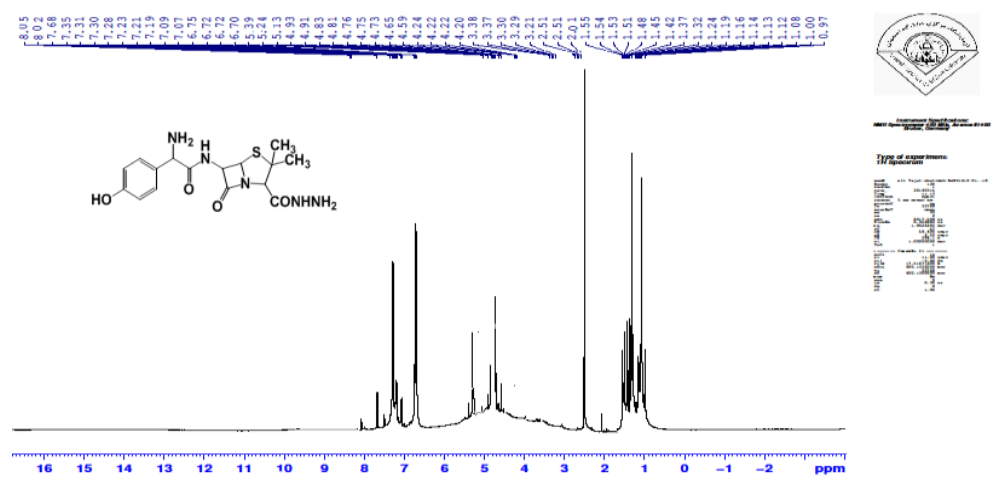

Figure.4 1HNMR Spectrum for Compound(4)

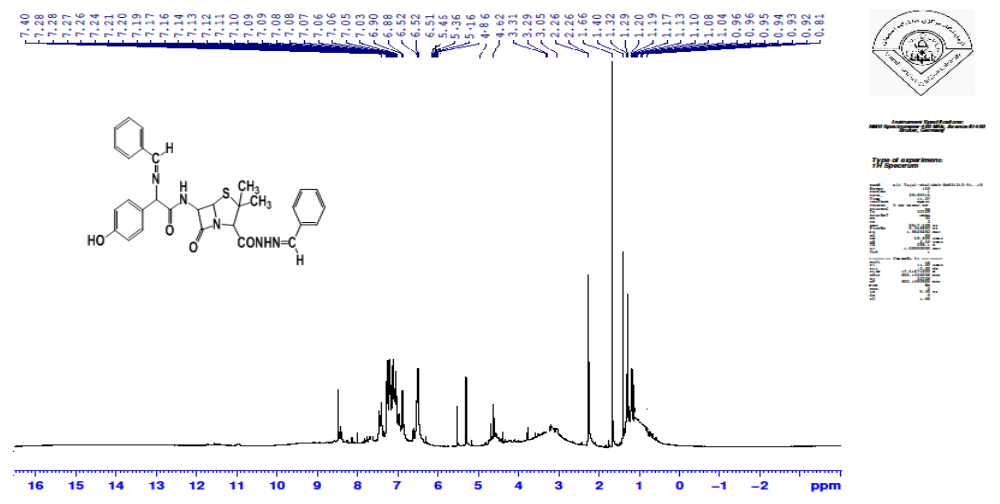


Figure.5 1HNMR Spectrum for Compound(5)

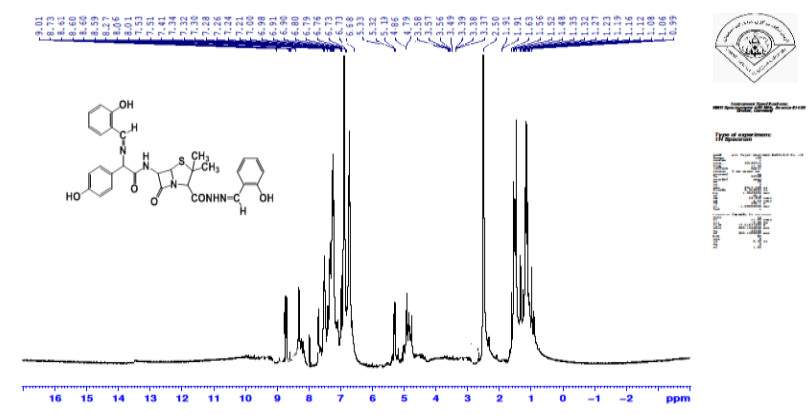

Figure.6 1HNMR Spectrum for Compound(9)

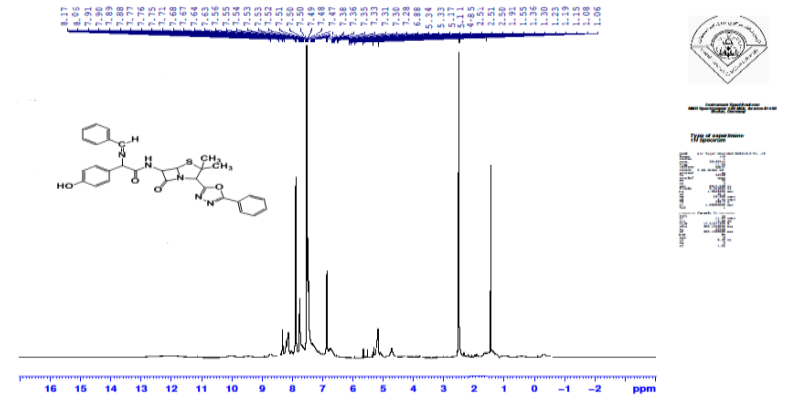

Figure.7 1HNMR Spectrum for Compound(10)

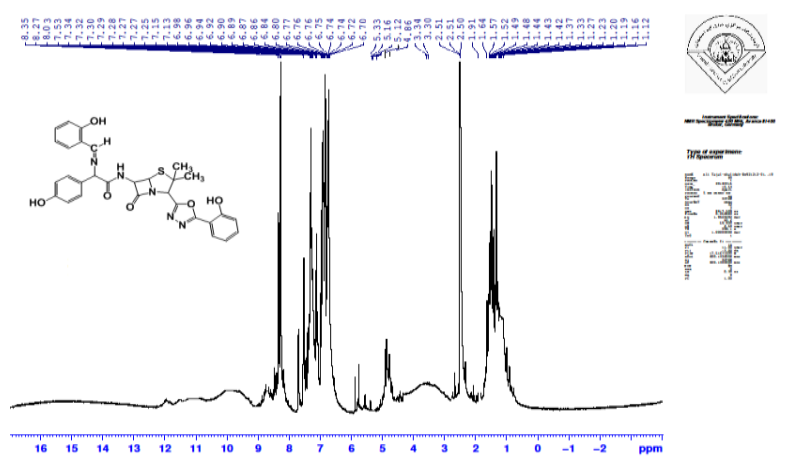

Figure.8 1HNMR Spectrum for Compound(14)

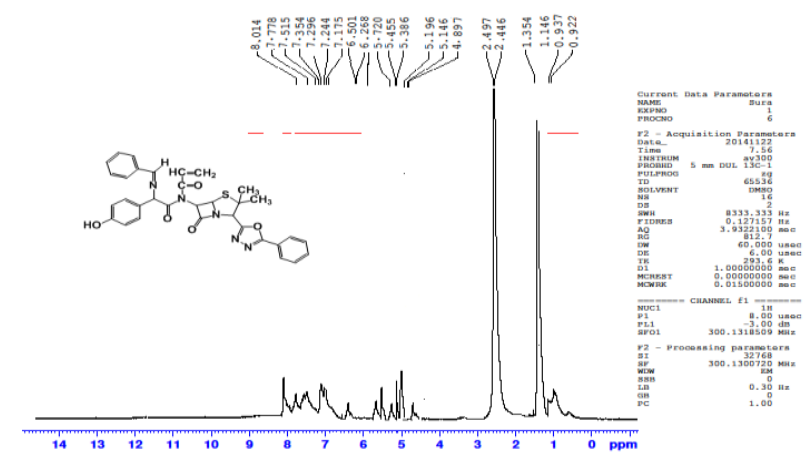


Figure.9 1HNMR Spectrum for Compound(19)

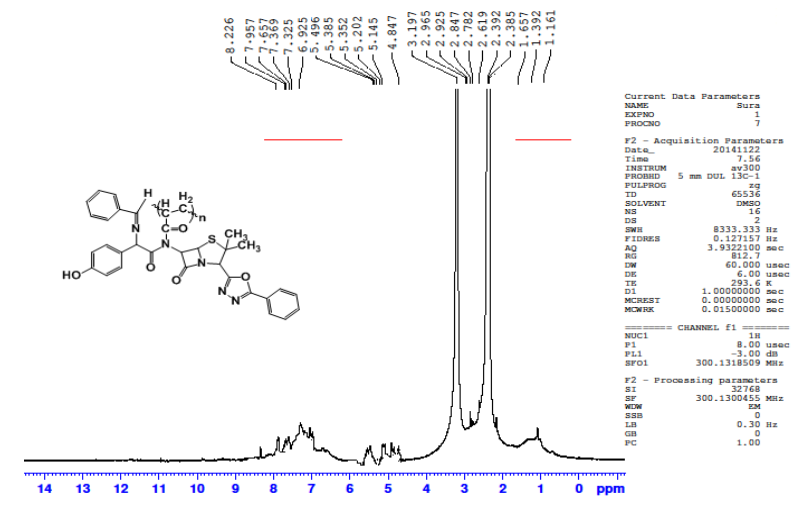

Scheme.1

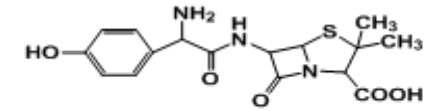

Amoxicillin (1)

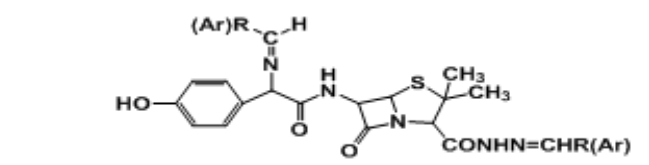

(4-8)

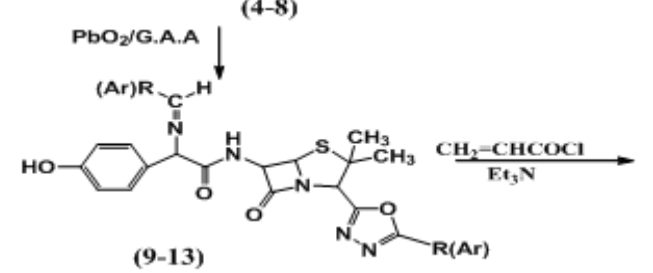

(9-13)

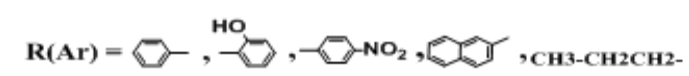

\section{Antibacterial Activity (22)}

The compounds (9-13) and compounds (1923) were evaluated their antibacterial activity in comparison to the control antibacterial drug amoxicillin against some bacterial species positive gram bacterial (Staphylococcus aureus and Bacillus) and negative gram bacterial (Escherichia coli and Pseudomonas aeruginosa) using DMSO as solvent to get desired concentration

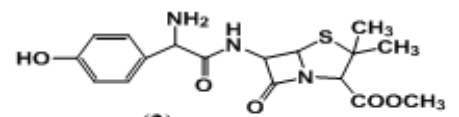

(2) $\mathrm{NH}_{2} \mathrm{NH}_{2} \cdot \mathrm{H}_{2} \mathrm{O}$

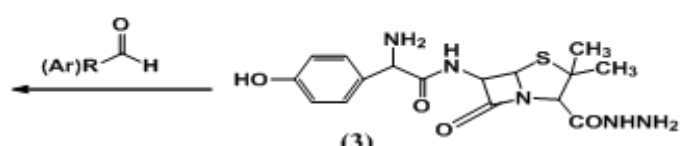

(3)
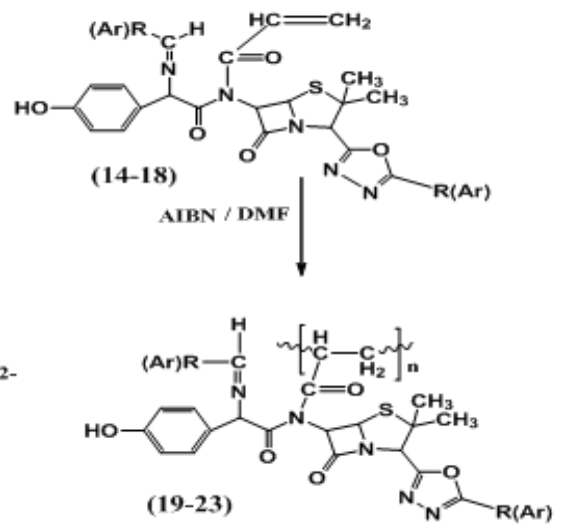

(1 $\mathrm{mg} / \mathrm{ml})$ by agar diffusion method, the plates were incubated at $37^{\circ} \mathrm{C}$ for 24 hours, the inhibition zone measured in $(\mathrm{mm})$. Synthesized compounds (9-13) were screened antibacterial activity and showed varying degree of inhibition zone against the tested gram positive and gram negative bacteria and observed that gram positive bacteria show better activity than gram negative bacteria, the compounds $(9,11$ and 13) show antibacterial activity against 
Pseudomonas aeruginosa compared with drugs that show no change in the growth of this bacteria. All compounds (19-23) showed antibacterial activity against the tested organisms. The compounds $(19,21)$ showed antibacterial activity better than drugs and other derivatives. The results of activity listed in table- 4 .

\section{Acknowledgement}

I would like to thank the Chemistry Department, College of Science for Women and Chemistry Department, College of Science, Baghdad University.and the College of Pharmacy AL-Mustansiriya University for their continual support throughout my experimental work.

\section{References}

1.S.Cledualdo; F. Bruno; M., Jose. Molecules 2012, 17, 10192-10231.

2.B. Holla; R. Gonsalves; S.Shenoy. Eur. J. Med. Chem., 2000,35,267-271.

3.E.Palaska; G. Sahin; P.Kelicen; N. Durlu. II Farmeco, 2002,57, 101-107.

4.Amir; K. Shikha.Eur. J. Med. Chem., 2004,39, 535-545.

5.J.,Bhat; B. Shah; H., Shah; P. Trivedi; N. Undavia. Indian.J. Chem, 1994, 33b, 189-192.

6.A.Zargahi; A. Sayyed; M. Tabatabai; A. Faizi; P. Ahadian; Navavi V., A. Zanganeh.Biorg. Med.Chem, 2005,. 15, 1863-1865.

7.M. Manav ;S. Mohit ;S. Abdul; D. Kash $J$. Serb. Chem. Soc., 2012, 77(2),9-16.
8.K. Mogilaiah;and N. Vasudeva. Indian Journal of Chemistry,2003., 42 B, 2124.

9.M. Chande; A. Godbole; E. Coutinho; P.Desai. Indian Journal of Chemistry, 2003, 42b, 397.

10.D. Dhansay; P. Alok. Int. J. Chem. Tech. Res.,2010, 2(3),1397-1412.

11.N. Milovic; J. Wang; K. Lewis; A. Klibanov. Biotechnol. Bioeng., 2005, 90,715-722.

12.A.Brar ; M. Malhotra. Macromolecules, 1996, 29,7470-7476.

13.S.Thamazharsi; A. Reddy. European Polymer Journal, 1992,28(2),119-123.

14.H. Omidin ; A. Hashemi; P. Sammes; G. Meldrum. Polymer, 1999, 40,1753-1761.

15.A.Lengu; D.Neckers. Journal of Coating Technology, 1995, 67,29-35.

16.D.Akash; P. Priyanka; K.Mahessh; K.Saloni.Acta Poloniae PharmaceuticaDrug Research, 2012, 69(1):129-133.

17.E.Shiva; R. Parameshwar ; V.Harinadha; Y.,Sri ; B.Kumar; G.Anil. Int J Pharm Pharm Sci 2012,4(1),424-427.

18.M.Akbar ; F.Naser ; K., Mansooreh ; E. Sattar. European Journal of Chemistry, 2010,1(1),33-36.

19.K.Palak; H. Parikh; M. Marvaniya ; J.Dhrubo. Int. J. Drug Dev. a nd Res., 2011, 3(2),248-255.

20.M. Khalid ;A. Ahmad; N. Amal. J. Edu. and Sci., 2009,22(3),11-18.

21.K.Hana; K. Ibtisam; A. Salwa. Journal of Al-Nahrain University, 2007,10(1),18-24.

22.M.Yar; M. Akhter.Acta. Pol. Pharm., 2009., 66(4),393-397.

\section{How to cite this article:}

Entesar, Obeed. AL-Tamimi and Khalida, Ali.Thejeel. 2016. Synthesis, Characterization and Polymerization of 1,3,4- Oxadiazole Derivatives of Amoxicillin and Evaluation Antibacterial Activities. Int.J.Curr.Microbiol.App.Sci.5(2): 511-522.

doi: http://dx.doi.org/10.20546/ijcmas.2016.502.058 